\title{
Results of the Optical Downlink Experiment KIODO from OICETS Satellite to Optical Ground Station Oberpfaffenhofen (OGS-OP)
}

\author{
N. Perlot ${ }^{a}$, M. Knapek ${ }^{a}$, D. Giggenbach ${ }^{a}$, J. Horwath ${ }^{a}$, M. Brechtelsbauer ${ }^{a}$, Y. Takayama $^{b}$, T. Jono ${ }^{b}$ \\ ${ }^{a}$ DLR, Institute of Communication and Navigation, 82234 Wessling, Germany \\ ${ }^{b}$ Japan Aerospace Exploration Agency, Tsukuba, Ibaraki, Japan
}

\begin{abstract}
Optical LEO downlinks from the Japanese OICETS to the optical ground station built by the German Aerospace Center (DLR) near Munich have been performed. This was the first optical LEO downlink on European grounds. The ground station received a 50-Mbit/s OOK signal at $847 \mathrm{~nm}$ on its $40-\mathrm{cm}$ Cassegrain telescope and sent two spatially displaced beacon beams towards OICETS. Five out of eight trials could be performed successfully while the other three were hindered by cloud blockage. A BER of $10^{-6}$ has been reached. The elevation angle above the horizon ranged between $2^{\circ}$ and $45^{\circ}$. The Fried parameter and the scintillation were measured with instruments inside the ground station. The beacon power received by the LUCE Terminal onboard OICETS has also been recorded. This paper describes the setup of the experiment and highlights the results of the measurement trials.
\end{abstract}

Keywords: OICETS, KIODO, free-space laser communications, scintillation

\section{INTRODUCTION}

The KIODO (KIrari's Optical Downlink to Oberpfaffenhofen) experiment took place in June 2006. An optical link was established between the OICETS satellite and a ground station located in Oberpfaffenhofen $(20 \mathrm{~km}$ south-west from Munich, Germany). OICETS is a LEO satellite owned by JAXA with a sun-synchronous orbit and an altitude of $610 \mathrm{~km}$. The KIODO experiment was performed a few months after the KODEN experiment [1] carried out with the NICT ground station in Japan. Table 1 provides an overview of the 8 trials that have been done during the KIODO experiment. A trial corresponds to a single particular pass of the OICETS satellite. This is because, for each trial, OICETS requires a special attitude configuration that must be planned some days in advance [2].

\begin{tabular}{|c|c|c|c|c|c|c|}
\hline $\begin{array}{c}\text { KIODO } \\
\text { Trial }\end{array}$ & $\begin{array}{c}\text { Day of } \\
\text { June } \\
2006\end{array}$ & $\begin{array}{c}\text { Time } \\
\text { (UTC) of } \\
\text { Pass Start }\end{array}$ & $\begin{array}{l}\text { Maximum } \\
\text { Pass } \\
\text { Elevation }\end{array}$ & $\begin{array}{c}\text { Measurement } \\
\text { Elevation Span }\end{array}$ & Weather & Remark \\
\hline KT 1 & $7^{\text {th }}$ & $1: 13$ & $45.5^{\circ}$ & $10-35^{\circ}$ & $90 \%$ clear sky, $8^{\circ} \mathrm{C}$ & $\begin{array}{c}\text { No scintillation / } \\
\text { DIMM measurement }\end{array}$ \\
\hline KT 2 & $9^{\text {th }}$ & 0:02 & $33.5^{\circ}$ & $10-32^{\circ}$ & $90 \%$ clear sky, $10^{\circ} \mathrm{C}$ & $\begin{array}{c}\text { No BER } \\
\text { measurement }\end{array}$ \\
\hline KT 3 & $14^{\text {th }}$ & $1: 04$ & $55.6^{\circ}$ & $10-45^{\circ}$ & clear sky, $10^{\circ} \mathrm{C}$ & \\
\hline KT 4 & $15^{\text {th }}$ & $23: 54$ & $28.0^{\circ}$ & $2-27^{\circ}$ & $80 \%$ clear sky, $12^{\circ} \mathrm{C}$ & \\
\hline KT 5 & $21^{\mathrm{st}}$ & $0: 56$ & $68.2^{\circ}$ & & Cloudy & No Link \\
\hline KT 6 & $23^{\text {rd }}$ & $1: 21$ & $38.4^{\circ}$ & & Cloudy & No Link \\
\hline KT 7 & $28^{\text {th }}$ & $0: 47$ & $83.2^{\circ}$ & $4-43^{\circ}$ & $\begin{array}{l}\text { Thunder clouds } 1 \mathrm{~h} \\
\text { before trial, } 18^{\circ} \mathrm{C}\end{array}$ & \\
\hline KT 8 & $30^{\text {th }}$ & $1: 12$ & $46.5^{\circ}$ & & Cloudy & No Link \\
\hline
\end{tabular}

Table 1 Overview of the KIODO trials. Local time is UTC +2 hours

(C) Copyright 2007 Society of Photo-Optical Instrumentation Engineers.

This paper was published in the Proceedings of SPIE Vol. 6457 and is made available as an electronic reprint with permission of SPIE. One print or electronic copy may be made for personal use only. Systematic or multiple reproduction, distribution to multiple locations via electronic or other means, duplication of any material in this paper for a fee or for commercial purposes, or modification of the content of the paper are prohibited. 
All trials occurred in the night at approximately the same local time. Viewed from the ground station, satellite passes have different maximum elevation angles. The operation of the optical terminal LUCE onboard OICETS had some constraints regarding its angular speed and the position of the sun with respect to its field of view. Due to these constraints, each trial was limited to a predetermined pass segment and a corresponding elevation span $\left(5^{\text {th }}\right.$ column in Table 1). To make comparisons easier in this paper, most results are shown as a function of the elevation angle. Because of cloud coverage, no link was established for the trials 5, 6 and 8.

\section{SCENARIO DESCRIPTION}

OICETS is optically compatible with the ARTEMIS satellite and therefore transmits data at $50 \mathrm{Mbit} / \mathrm{s}$ with an OOK modulation and at a wavelength of $847 \mathrm{~nm}$ [3] and receives beams with wavelengths near $810 \mathrm{~nm}$. As depicted in Fig. 1, the KIODO downlink involves two beams: the beacon beam from the ground station (wavelength of $808 \mathrm{~nm}$, divergence of $5 \mathrm{mrad}$ ) and the communication beam from OICETS (divergence of $5 \mu \mathrm{rad}[3]$ ).

The ground station is a clamshell dome of 4-m diameter installed on a building's roof. The terminal, which consists of a 40-cm cassegrain telescope supported by an astronomical mount, is transportable [4]. The telescope and the mount can be seen in Fig. 2. The communication receiver, the tracking camera, the measurement cameras and a powermeter are located on a metal plate fixed behind the telescope. The beacon beam was transmitted by two spatially displaced 5-cm telescopes mounted on each side of the 40-cm telescope.

Before the start of a trial, the ground station knows precisely OICETS' orbit data and points its beacon towards the satellite. On reception of the beacon, LUCE sends its communication beam towards the ground terminal. At the ground station, the beam is acquired on the acquisition camera (wide field of view) and tracking camera (narrow field of view), and a closed-loop tracking keeps the pointing error below $100 \mu \mathrm{rad}$. The pointing error during KT3 can be seen at the top of Fig. 3. The power received over the $40-\mathrm{cm}$ telescope during KT3 is plotted in Fig. 4. The periodical signal breaks are characteristic of the LUCE terminal [2] and appeared in each link performed.

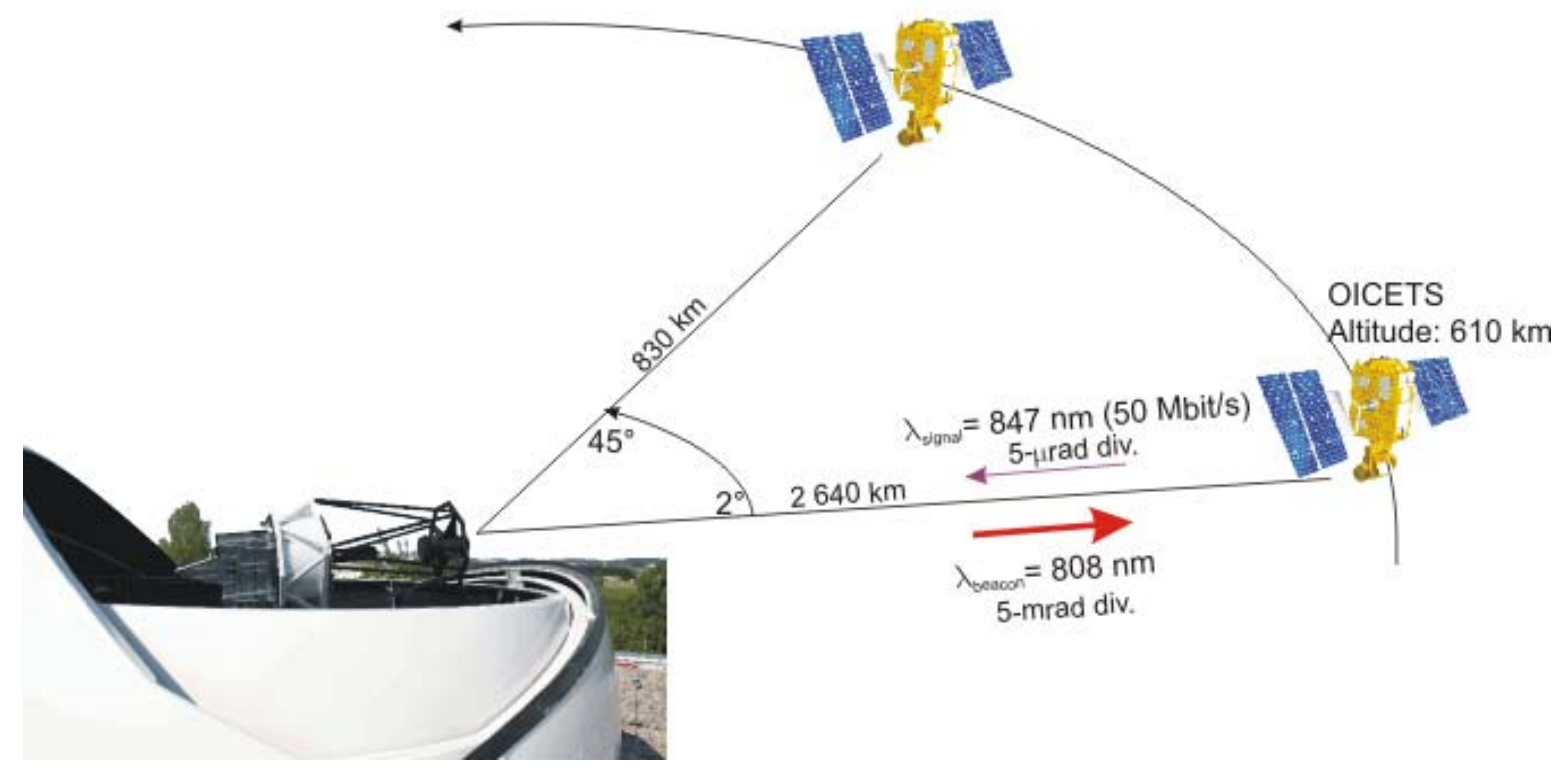

Fig. 1 Overview of the link configuration. 


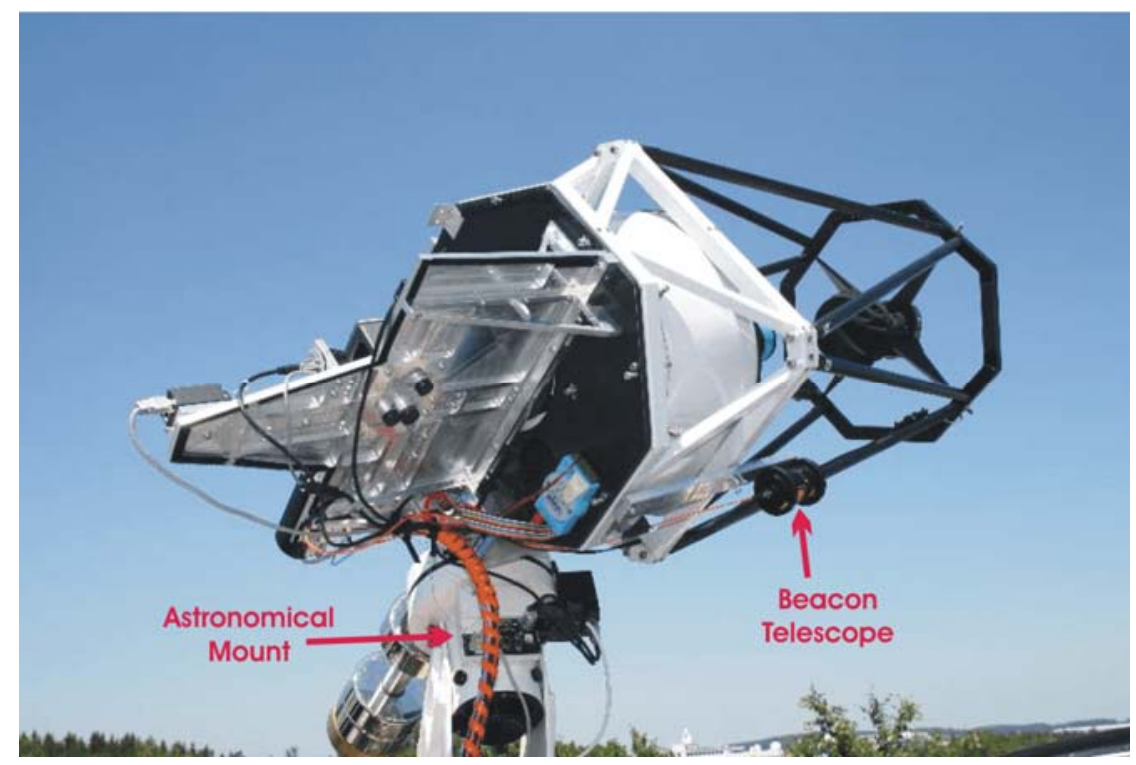

Fig. 2 Rear view of the $40-\mathrm{cm}$ telescope.


Fig. 3 Ground-station pointing error (uppermost plot) and pass parameters (lower plots) during KT3 as a function of time. 


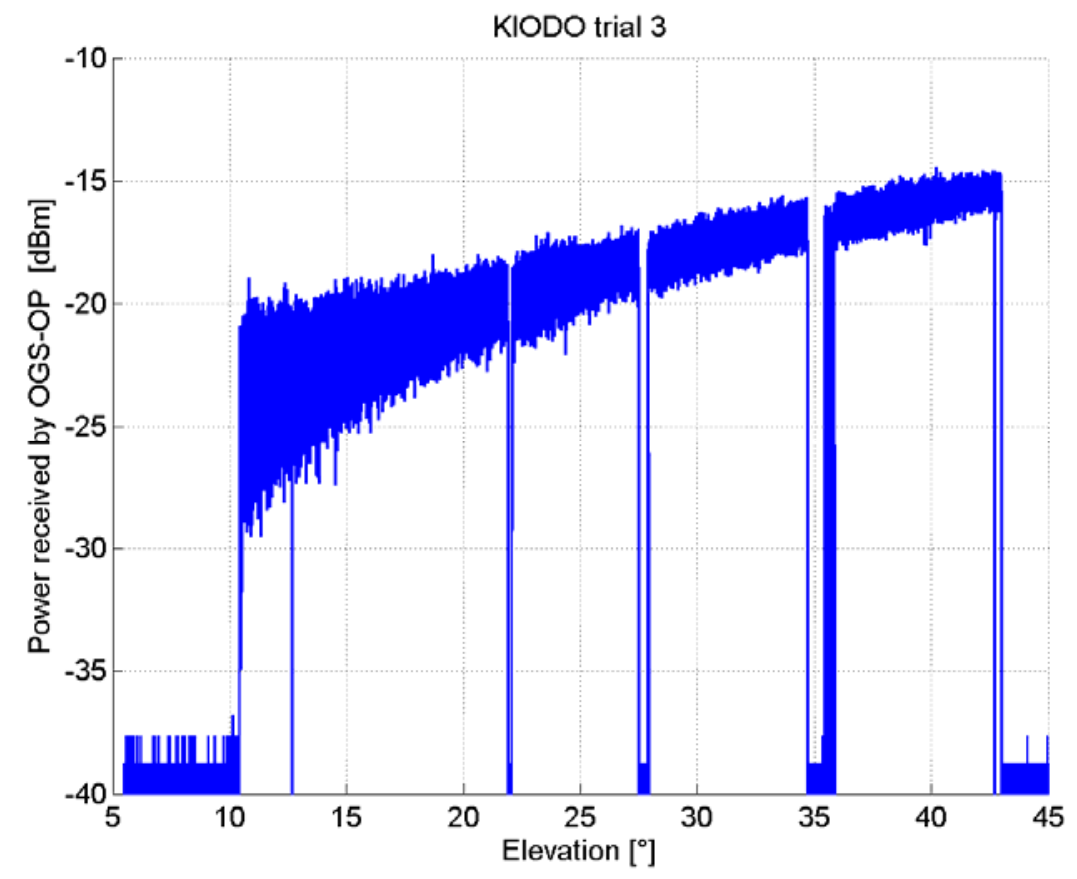

Fig. 4 Optical Power impinging on the 40-cm telescope in KT3.

\section{BEACON UPLINK}

Because the optical-power acceptance range of OICETS' sensors is small, the beacon transmit power was adjusted as a function of the elevation angle. Fig. 5 shows how the power has been decreased with the elevation in order to keep constant the mean optical power on the LUCE terminal. The clear-sky extinction coefficient was evaluated as a function of altitude for a wavelength $\lambda=810 \mathrm{~nm}$ [5]. Fig. 6 shows the power received by LUCE during KT3. The scintillation of the beacon beam was reduced by transmitting it as a spatially incoherent beam. The separation distance between the two beacon apertures was about $50 \mathrm{~cm}$. The beacon beam was a multimode beam coming from two 400- $\mu \mathrm{m}$ multimode fibers. Fig. 7 shows the scintillation index (normalized variance of optical power) measured by the LUCE terminal. Two effects have further reduced the scintillation as plotted in Fig. 7. First, temporal averaging has occurred due to LUCE's 1$\mathrm{kHz}$ sampling rate. Second, at low elevations some high power values have saturated on LUCE's sensors.

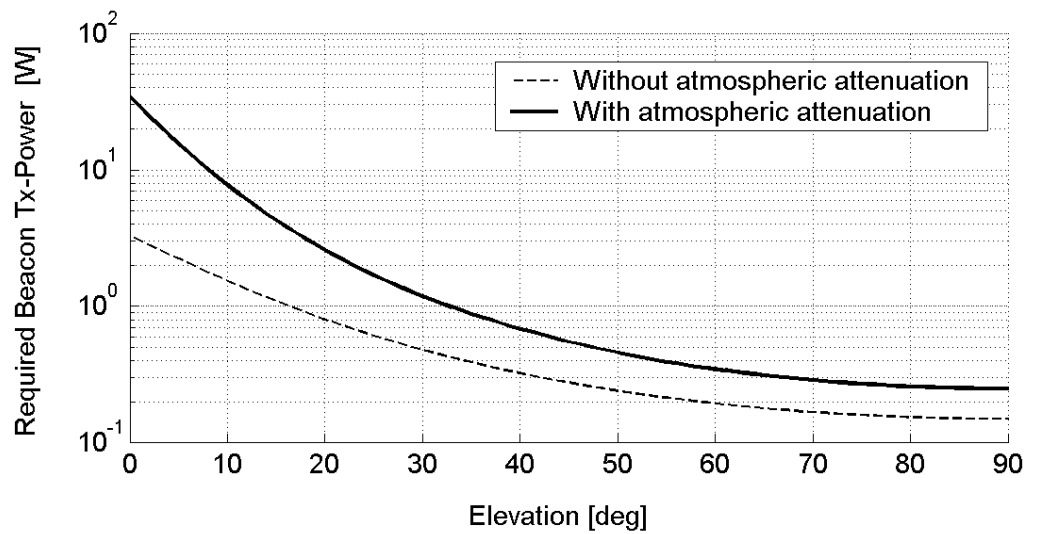

Fig. 5 Calculated level of the total transmitted beacon output power in order to obtain the desired power level on OICETS. 


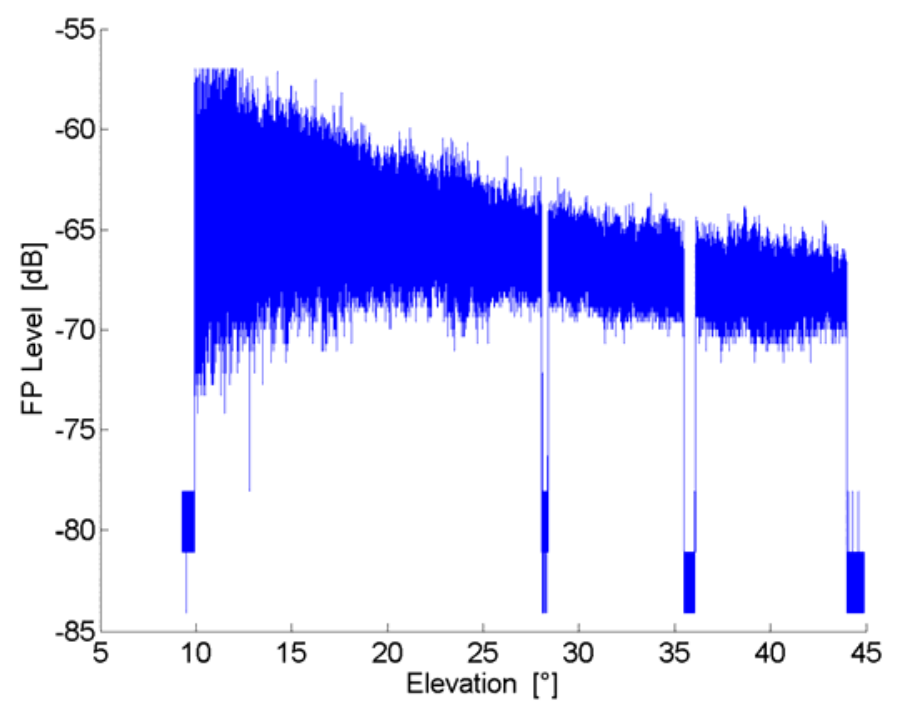

Fig. 6. Optical power measured by LUCE's fine pointing (FP) sensors during KT3.

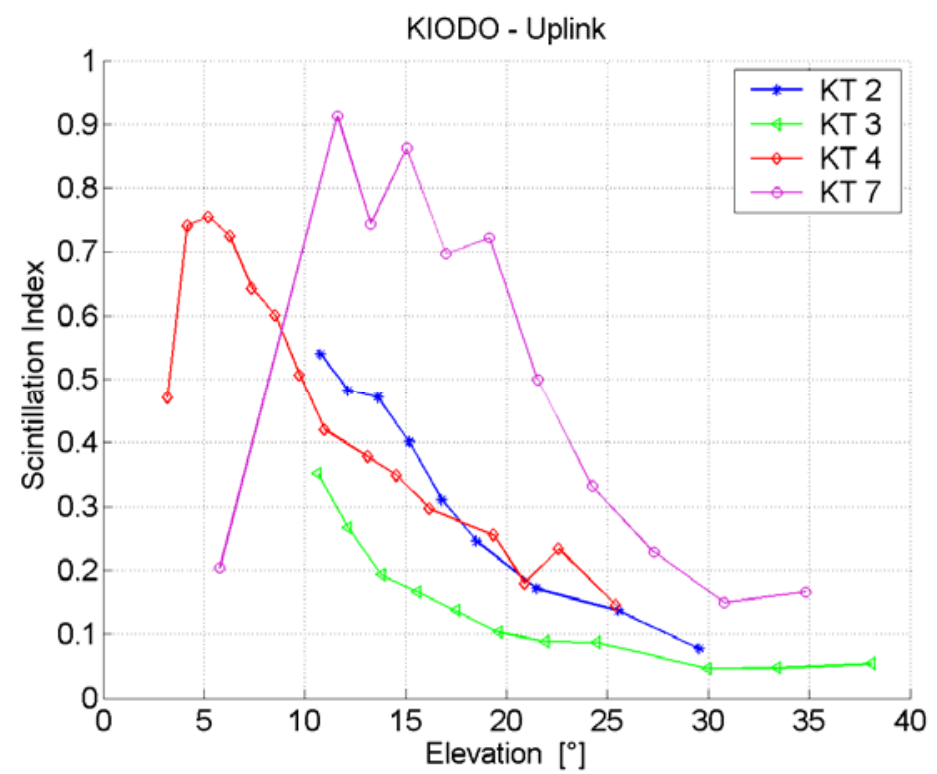

Fig. 7 Scintillation of the beacon beam at the satellite. Sampling rate is $1 \mathrm{kHz}$. Under-estimation occurs at low elevations due to sensor saturation of high received power values.

\section{DOWNLINK}

The downlink signal was a pseudo-random bit sequence at a rate of $50 \mathrm{Mbit} / \mathrm{s}$. The BER estimated twice per second is shown in Fig. 8. The BER decreases with elevation as a result of an increase of the mean power and a decrease of scintillation. The best achieved BER is $2 \times 10^{-6}$.

Fig. 9 is a spectrogram of the power received by the 40-cm telescope ground station during KT3. The cut-off frequency does not vary much over the considered elevation range and remains around $500 \mathrm{~Hz}$. Note that the cut-off frequency of scintillation over a smaller receiving aperture would have been slightly higher [6]. The temporal spectrum 
is affected by the motion of the beam through turbulent eddies. Viewed from the ground station, the angular speed of the satellite increases with the elevation and also with the maximum elevation of the pass. Fig. 10 shows the slew rate of the beam for passes with different elevation maxima.

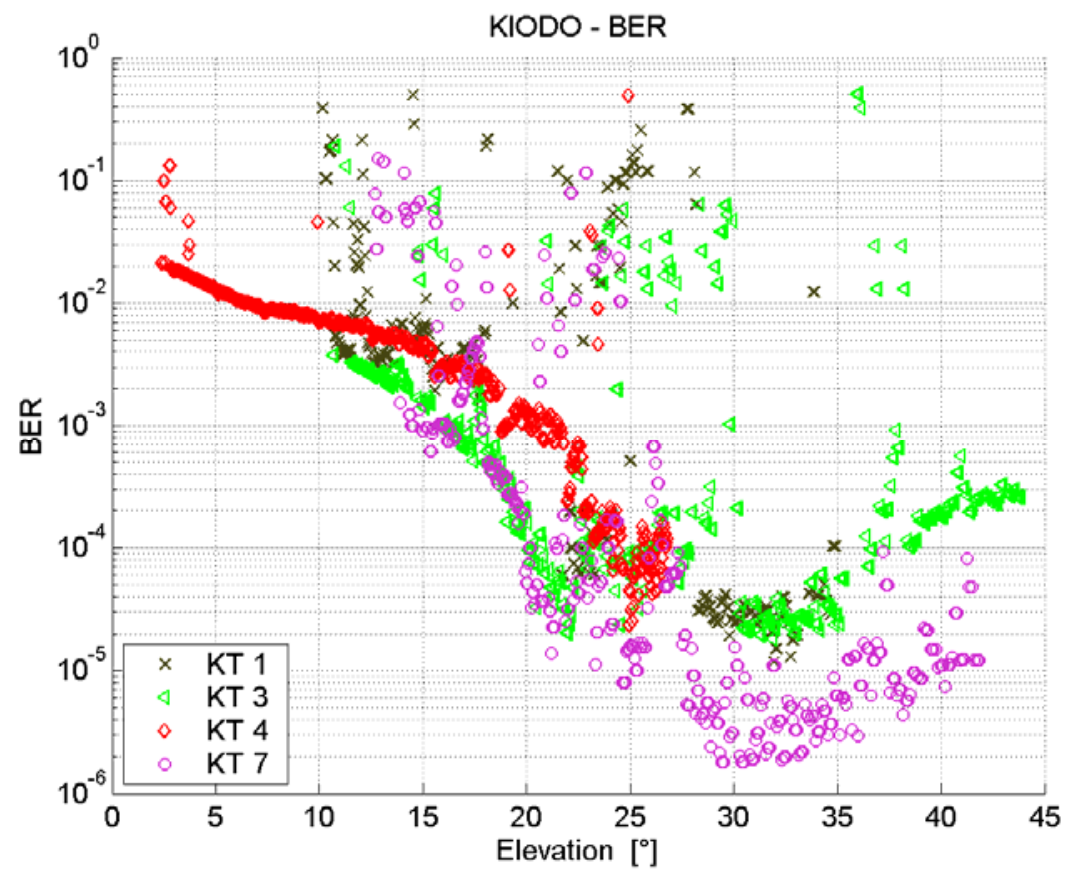

Fig. 8 BER measured during the KIODO trials

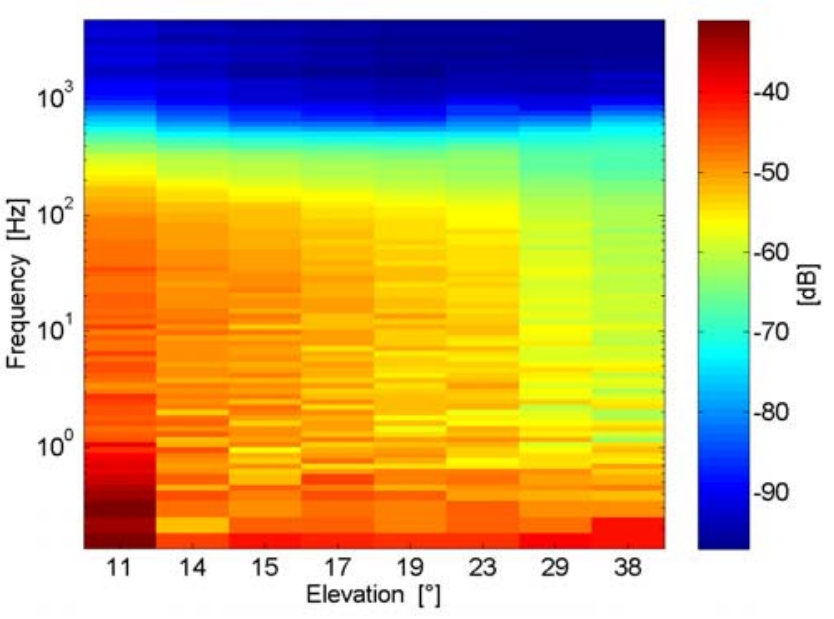

Fig. 9 Spectrogram of the optical power received by the 40$\mathrm{cm}$ telescope during KT3.

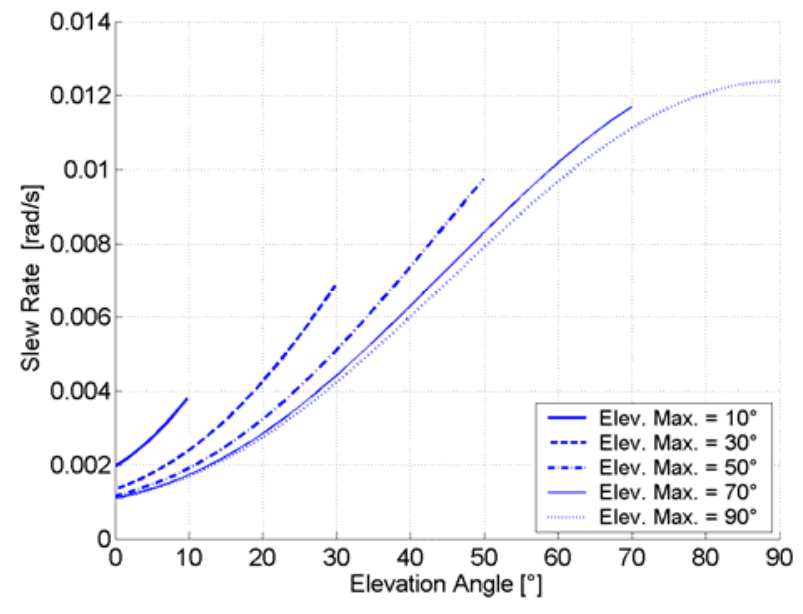

Fig. 10 Slew rate of the beam during links of OICETS (circular orbit at $610-\mathrm{km}$ altitude) with ground. The different types of satellite passes are identified by their elevation maxima.

On a first camera, pupil images have been recorded to measure scintillation. On a second camera, the Fried parameter $r_{0}$ was estimated using the DIMM (Differential Image Motion Monitor) concept. Both cameras had an exposure time of $80 \mu \mathrm{s}$ which is short enough to "freeze" the images in time. 
The scintillation index of the intensity (i.e., of a camera pixel) is shown in Fig. 11 as a function of elevation. The scintillation dynamic was fully rendered by the 12-bit resolution of the camera. As predicted by theory [6], we observe saturation of scintillation at low elevations. Also interesting is the large variation of the scintillation between two different nights: scintillation during KT7 was 5 times stronger than during KT3. The main difference between the two trials is the presence of a warm thunderstorm a few hours before KT7. The intensity spatial covariance was also estimated from the speckle images and the associated correlation length is shown in Fig. 12. Over the aperture of $40-\mathrm{cm}$ diameter (secondary-mirror obscuration of 12-cm diameter), the aperture averaging factor was approximately 0.2 and 0.1 at $5^{\circ}$ and $30^{\circ}$ elevation, respectively. DIMM results are displayed in Fig. 13. We have used the standard DIMM procedure [7] in which the focal-spot motion variance is related to $r_{0}$ assuming Kolmogorov wavefront distortions and no scintillation. Note that these assumptions may have a decreased validity for strong-fluctuation measurements.

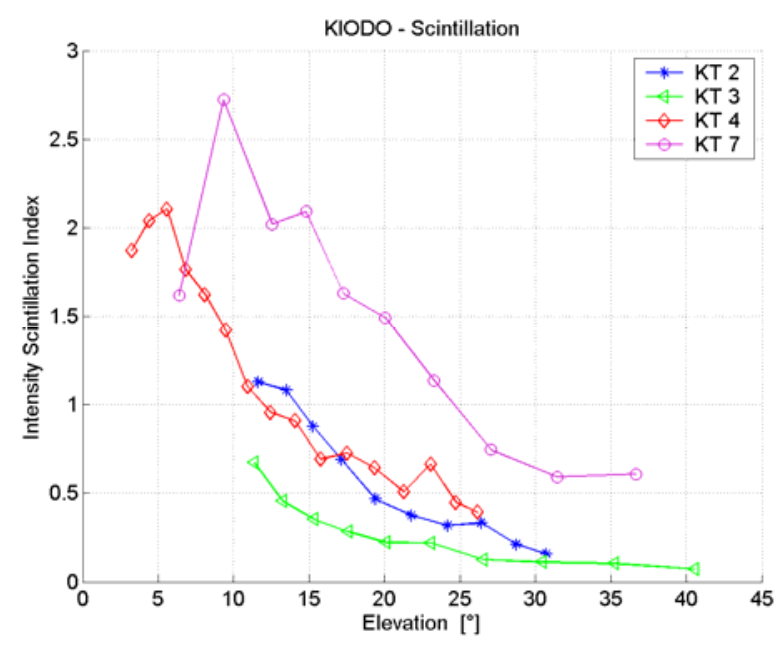

Fig. 11 Intensity scintillation index of the downlink.



Fig. 12 Intensity correlation length as determined by the $1 / \mathrm{e}$ point of the estimated intensity covariance function.

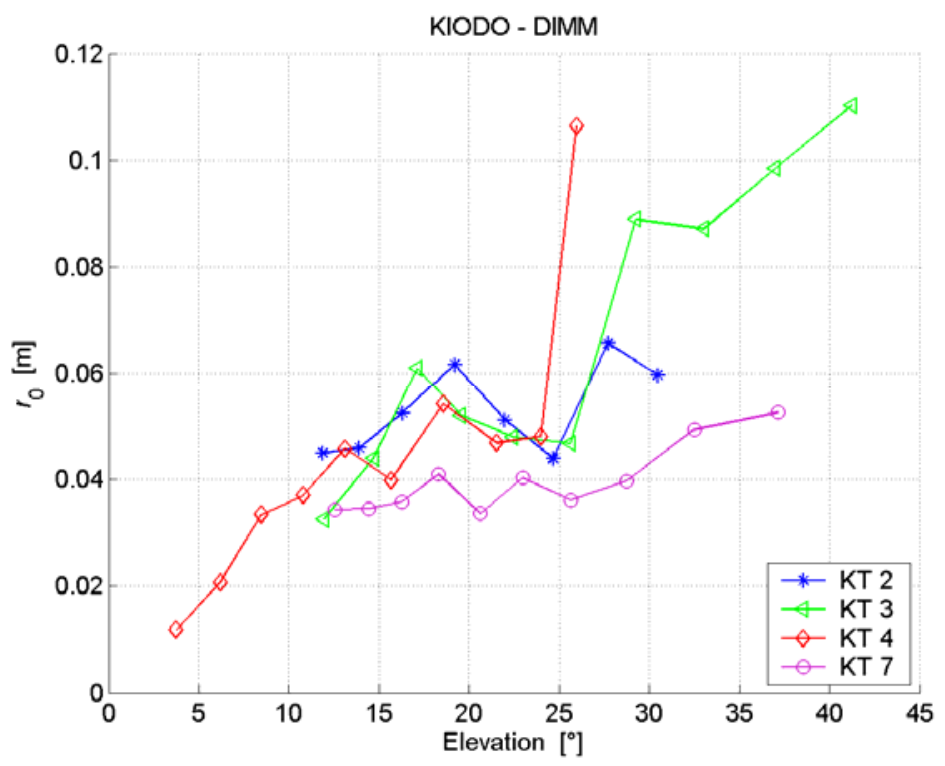

Fig. $13 r_{0}$ measurements from the DIMM instrument. 


\section{CONCLUSIONS}

A simple optical ground terminal has performed an optical downlink with the LEO satellite OICETS with BERs as low as $2 * 10^{-6}$. It is believed that a receiving front-end more robust against scintillation would have achieved an error-free data transmission. We observed a general agreement of measurements with scintillation theory. Measurements at low elevations have enabled the observation of scintillation saturation. This successful experiment encourages to further pursue the concept of direct optical high-speed downlinks from earth observation satellites. DLR's Optical Communication Group (OCG) thanks JAXA's OICETS-team for their cooperation and M. Toyoshima from NICT for his help.

\section{REFERENCES}

[1] M. Toyoshima, K. Takizawa, T. Kuri, W. Klaus, M. Toyoda, H. Kunimori, T. Jono, Y. Takayama, N. Kura, K. Ohinata, K. Arai and K. Shiratama, "Ground-to-OICETS laser communication experiments", Proc. of SPIE, 6304B, 1-8 (2006)

[2] Y. Takayama, T. Jono, M. Toyoshima, H. Kunimori, , D. Giggenbach, N. Perlot, M. Knapek, K. Shiratama, J. Abe, K. Arai, Tracking and pointing characteristics of OICETS optical terminal in communication demonstrations with ground stations, Free-Space Laser Communication Technologies XIX, Proc. of SPIE, 6457A (2007).

[3] T. Jono, Y. Takayama, N. Kura, K. Ohinata, Y. Koyama, K. Shiratama, Z. Sodnik, B. Demelenne, A. Bird and K. Arai, "OICETS on-orbit laser communication experiments", Proc. of SPIE, 6105, 610503, 1-11 (2006).

[4] M. Knapek, J. Horwath, N. Perlot, K. Zettl, D. Giggenbach, B. Wilkerson, "Transportable Optical Ground Station for Free-Space Laser Communications", 3rd Advanced Satellite Mobile Systems Conference (ASMS), Herrsching (2006)

[5] B. Mayer, S. Shabdanov, D. Giggenbach, "Electronic Database of atmospheric absorption coefficients", DLRinternal report by DLR-IPA and DLR-IKN-DN-OCG, DLR-Oberpfaffenhofen (2002), based on the atmospheric constituents profiles according to "G.P. Anderson, et al: AFGL Atmospheric Constituent Profiles (0-120km), AFGL-TR-86-0110, Hanscom Air Force Base, MA 01736 (1986)"

[6] L. C. Andrews, R. L. Phillips, Laser Beam Propagation through Random Media, $2^{\text {nd }}$ Edition, SPIE Press, Bellingham, (2005)

[7] M. Sarazin, F. Roddier, "The ESO differential image motion monitor", Astronomy \& Astrophysics, 227, 294300 (1990). 